Falk Uebernickel, Axel Hochstein, Veit Schulz, Walter Brenner

\title{
Excellence-Modell der Industrialisierung des Informationsmanagements
}

Die Akzeptanz der Dienstleistungen von IT-Einheiten in Industrie und Verwaltung ist trotz allen Fortschritts in Technik und Prozessen nicht zufriedenstellend. Das industrialisierte Informationsmanagement (IIM) will hier durch eine neue Ausrichtung der Methoden und Inhalte des IT-Managements helfen, die IT ihrer Bedeutung entsprechend zu managen. Der Beitrag beschäftigt sich mit der Entwicklung eines ExcellenceModells zur Bewertung und Einschätzung des Industrialisierungsgrades eines IT-Dienstleisters. Zur Strukturierung der Analyse gruppiert das Modell die Aufgaben eines IT-Dienstleisters in 12 Rollen, wie z. B. Produkt-Engineering, Sourcing, Delivery-Management, Controlling, und bildet drei Analyseebenen: die genannten Rollen, darüber Prozesse und schließlich das Gesamtunternehmen. Am Praxisbeispiel eines deutschen Unternehmens wird das Excellence-Modell für die Rolle Controlling beschrieben. Erste Erkenntnisse der Anwendung zeigen auf, zu welchen Anregungen die Nutzung des Modells im Beispielunternehmen geführt hat.

\section{Inhaltsübersicht}

1 Ausgangssituation und Zielsetzung

2 Reifegradmodelle im Überblick

3 Grundlagen des IIM-Excellence-Modells

3.1 Konzepte des Excellence-Modells

3.2 Analyseebenen

3.3 Ergebnisraum

4 Aufbau des IIM-Excellence-Modells

4.1 Kategorien der Excellence

4.2 Stufen der Industrialisierung

4.3 Dimensionen der Analyseelemente

5 Analyseelemente am Beispiel einer ausgewählten Rolle

5.1 Messkriterien der Industrialisierung am Beispiel des IT-Controllings

5.2 Praxisbeispiel

6 Literatur

\section{Ausgangssituation und Zielsetzung}

Die allgemeinen Prinzipien der Industrialisierung - wie Produkt- und Prozessstandardisierung, Automatisierung, Prozesstransparenz und -effizienz, Reduktion der Wertschöpfungstiefe, Modularisierung, Flexibilisierung und Mass Customization - finden auch bei IT-Dienstleistern zunehmend Geltung und erzeugen dadurch eine ähnliche Professionalisierung und Effizienzsteigerung, wie sie im letzten Jahrhundert in traditionellen Branchen erfolgt sind [Hochstein 2006; Hochstein et al. 2006]. Hierzu befähigt werden IT-Organisationen z. B. durch:

- IT-Prozessstandards (z. B. ITIL, CobiT, eTOM oder (MMI),

- IT-Standardprodukte (z. B. On-Demand-Software oder IT-Commodities im ERP-Umfeld),

- Virtualisierungstechnologien (z. B. Enterprise Service Bus, Grid Computing oder Integrationsplattformen wie den SAP Netweaver oder IBM Websphere),

- technologische Standards (z. B. neue Protokolle wie SOAP oder Ajax und neue Sprachen wie XML, WSDL oder BPELWWS) und

- neue Architekturen und die damit verbundene, veränderte Art und Weise der Softwareentwicklung (z.B. SOA, Composed Services oder Mashups).

Wie industrialisiert und damit professionell sind jedoch derzeit einzelne IT-Organisationen und der Branchendurchschnitt? Um hierzu eine Aussage treffen zu können, ist es notwendig, einzelne Organisationen bezüglich ihres Industrialisierungsgrades zu analysieren, zu bewerten und einen Branchendurchschnitt zu bestimmen. Dabei stellt sich die Frage, wie der Grad der Industrialisierung einer Organisation be- 
stimmt werden kann. In anderen Bereichen werden hierzu üblicherweise Reifegradmodelle verwendet. So wird für den Reifegrad der Softwareentwicklung beispielsweise das CMMI genutzt. Derartige Reifegradmodelle ermöglichen es Unternehmen, sich hinsichtlich bestimmter Zielstellungen zu analysieren, zu bewerten und gegebenenfalls Handlungsmaßnahmen für eine Verbesserung abzuleiten. Vor diesem Hintergrund ist es Ziel dieses Beitrags, ein Excellence-Modell für die IT-Industrialisierung zu entwickeln und zu beschreiben.

Die in diesem Beitrag präsentierten Ergebnisse sind im Rahmen einer seit fünf Jahren bestehenden, industriefinanzierten Forschungsgruppe entstanden. Dabei wurden die Ergebnisse auf Basis von Experteninterviews, Fallstudien [Yin 2002] sowie Fokusgruppen [Greenbaum 1988] etappenweise erarbeitet. Unter anderem wurden auch die Anforderungen, die an ein Modell zur Bestimmung des Industrialisierungsgrades zu stellen sind, in dieser Forschungsgruppe ermittelt:

\section{Berücksichtigung der gesamten IT-Wertkette}

Um eine umfassende Bewertung der IT-Industrialisierung vornehmen zu können, genügt es nicht nur einen Ausschnitt der Managementbereiche eines IT-Dienstleisters zu betrachten. Ein alleiniger Fokus auf die Softwareentwicklung, auf den IT-Betrieb oder die IT-Governance würde keine umfassende Sicht garantieren. Nur ein wertkettenorientierter Ansatz sichert die Berücksichtigung aller funktionalen Bereiche der IT-Leistungserstellung und deren Unterstützungsprozesse.

\section{Berücksichtigung des spezifischen}

Unternehmenskontexts und Integration von Handlungsmaßnahmen

Des Weiteren muss das konzipierte Modell den spezifischen Unternehmenskontext einer IT-Organisation in der Bewertung mit berücksichtigen. Konkret müssen dabei Kriterien zur Einordnung der Organisation bereitgestellt und je nach Einordnung objektive Elemente zur Bestimmung des Industrialisierungsgrades an die Hand gegeben werden. Dadurch wird auf der einen Seite der Unternehmenskontext berücksichtigt und auf der anderen Seite die Objektivität und damit Vergleichbarkeit sichergestellt.

\section{Reifegradmodelle im Überblick}

Den Ausgangspunkt für die Entwicklung eines Excellence-Modells im Bereich der IT-Industrialisierung stellt die Untersuchung der bereits bestehenden Ansätze dar. Dazu wurden viele Prozessmodelle aus dem IT-Bereich anhand der Eigenschaften Verbreitungsgrad in der Praxis, abgedeckter Bereich und Struktur näher analysiert und verglichen. Ein strukturierter Auszug aus der umfangreichen Analyse findet sich in Tabelle 1.

Ausgehend davon konnten Schwachstellen hinsichtlich der Erfüllung der in Kapitel 1 beschriebenen Anforderungen an ein ExcellenceModell für die IT-Industrialisierung abgeleitet werden.

Wie bereits erwähnt, erfordert eine Begutachtung der Industrialisierung eines IT-Dienstleisters die Betrachtung der gesamten Wertkette. Die existierenden Modelle fokussieren aber schwerpunktmäßig nur auf Teilbereiche, so zum Beispiel auf die IT-Governance bei CobiT. Da jedoch die anderen Bereiche nicht oder kaum Gegenstand der Untersuchung sind, finden keineswegs alle Prozesse und Aufgaben eines IT-Service-Providers Beachtung. Nur eine ganzheitliche Betrachtung lässt jedoch ein Urteil hinsichtlich der Industrialisierung der Organisation zu. So sind beispielsweise im Rahmen einer integrierten Leistungserstellung intensive Abstimmungen zwischen Entwicklungsmanagement, Portfoliomanagement und dem Management der Produktion bedeutend und müssen sich in einem Modell wiederfinden [Zarnekow et al. 2006].

Ein weiterer wichtiger Aspekt ist, dass bei keinem dieser Modelle der Grundgedanke der 


\begin{tabular}{|c|c|c|c|c|c|}
\hline Modelle & $\begin{array}{l}\text { BS } 15000 \\
\text { [BSI 2002] }\end{array}$ & $\begin{array}{l}\text { CMMI Develop- } \\
\text { ment } 1.2 \\
\text { [SEI 2006] }\end{array}$ & $\begin{array}{l}\text { IT Service CMM } \\
\text { [Niessink } \\
\text { et al. 2005] }\end{array}$ & $\begin{array}{l}\text { eSCM } \\
\text { [Hyder et al. 2004] }\end{array}$ & $\begin{array}{l}\text { CobiT } \\
\text { [ISACA 2005] }\end{array}$ \\
\hline Bereich & $\begin{array}{l}\text { IT-Service- } \\
\text { management }\end{array}$ & $\begin{array}{l}\text { - Projekt- und } \\
\text { Prozessmanage- } \\
\text { ment, System- } \\
\text { Engineering, } \\
\text { Hardware und } \\
\text { Software } \\
\text { Engineering, } \\
\text { Supporting- } \\
\text { Prozesse }\end{array}$ & $\begin{array}{l}\text { - alle Aktivitäten } \\
\text { beim Service- } \\
\text { Delivery-Prozess } \\
\text { - IT-Service-Provi- } \\
\text { der }\end{array}$ & $\begin{array}{l}\text { - Management } \\
\text { von Sourcing- } \\
\text { Beziehungen } \\
\text { zwischen Liefe- } \\
\text { ranten und } \\
\text { Kunden } \\
\text { - IT-Service- } \\
\text { Provider }\end{array}$ & $\begin{array}{l}\text { - IT-Governance } \\
\text { - Entwicklung von } \\
\text { Richtlinien und } \\
\text { Good Practices } \\
\text { für die Steue- } \\
\text { rung der IT über } \\
\text { das gesamte } \\
\text { Unternehmen }\end{array}$ \\
\hline Zweck & $\begin{array}{l}\text { Best-Practices- } \\
\text { Anleitung für Orga- } \\
\text { nisationen für Sup- } \\
\text { port und Lieferung } \\
\text { von Services, die } \\
\text { auf Informations- } \\
\text { und Kommunika- } \\
\text { tionstechnologie } \\
\text { basieren } \\
\text { - Benchmarking }\end{array}$ & $\begin{array}{l}\text { - Verbesserung } \\
\text { der Entwick- } \\
\text { lungs- und War- } \\
\text { tungsprozesse } \\
\text { für Produkte und } \\
\text { Dienstleistungen } \\
\text { - Sammlung von } \\
\text { Best Practices }\end{array}$ & $\begin{array}{l}\text { Bewertung und } \\
\text { Verbesserung } \\
\text { der Fähigkeiten } \\
\text { eines IT-Service- } \\
\text { Providers bzgl. } \\
\text { Service Delivery }\end{array}$ & $\begin{array}{l}\text { - Bewertung und } \\
\text { Verbesserung } \\
\text { der Fähigkeiten, } \\
\text { hoch qualitative } \\
\text { Sourcing-Ser- } \\
\text { vices zu liefern } \\
\text { - Differenzierung } \\
\text { vom Wettbe- } \\
\text { werber }\end{array}$ & $\begin{array}{l}\text { - Überprüfung und } \\
\text { Kontrolle von } \\
\text { Informations- } \\
\text { systemen } \\
\text { - IT-Ausrichtung } \\
\text { und Governance }\end{array}$ \\
\hline Struktur & $\begin{array}{l}\text { - } 13 \text { Service- } \\
\text { management- } \\
\text { Prozesse (unterteilt } \\
\text { in } 5 \text { verschiedene } \\
\text { Bereiche) } \\
\text { - } 3 \text { weitere, überge- } \\
\text { ordnete Bereiche } \\
\text { (Policies, Frame- } \\
\text { work, Ziele, Pla- } \\
\text { nung) }\end{array}$ & $\begin{array}{l}\text { - } \text { Reifegradmodell } \\
\text { - } 22 \text { Process } \\
\text { Areas } \\
\text { - in } 4 \text { Kategorien } \\
\text { eingeteilt } \\
\text { - } 2 \text { Darstellungen: } \\
\text { kontinuierlich } \\
\text { und gestaffelt } \\
\text { - } 6 \text { Fähigkeits- } \\
\text { grade } \\
\text { - } 5 \text { Reifegrade }\end{array}$ & $\begin{array}{l}\text { - Reifegradmodell } \\
\text { - } 5 \text { Reifegrade } \\
\text { - } 22 \text { Key Process } \\
\text { Areas, jeweils } \\
\text { bestimmten Rei- } \\
\text { fegraden zuge- } \\
\text { ordnet } \\
\text { - für jede Key } \\
\text { Process Area: } \\
5 \text { Common } \\
\text { Features }\end{array}$ & $\begin{array}{l}\text { - Capability- } \\
\text { Modell (5 Fähig- } \\
\text { keitslevel) } \\
\text { - Sourcing- } \\
\text { Lebenszyklus } \\
\text { (4 Phasen) } \\
\text { - } 10 \text { Capability } \\
\text { Areas } \\
\text { - } 84 \text { Praktiken }\end{array}$ & $\begin{array}{l}\text { - } 4 \text { Domänen } \\
\text { - } 34 \text { zentrale } \\
\text { IT-Prozesse } \\
\text { - Kontrollziele } \\
\text { - } \text { Reifegradmodell } \\
\text { (6 Stufen) }\end{array}$ \\
\hline Entwickler & $\begin{array}{l}\text { BSI (British Stan- } \\
\text { dards Institution) }\end{array}$ & $\begin{array}{l}\text { SEl (Software } \\
\text { Engineering Ins- } \\
\text { titute, Carnegie } \\
\text { Mellon) }\end{array}$ & $\begin{array}{l}\text { - Projekte versch. } \\
\text { niederländi- } \\
\text { scher Universi- } \\
\text { täten mit Praxis- } \\
\text { partnern }\end{array}$ & - ITsqc & $\begin{array}{l}\text { - ITGI (IT Gover- } \\
\text { nance Institute) }\end{array}$ \\
\hline
\end{tabular}

Tab. 1: IT-Prozessmodelle

IT-Industrialisierung im Vordergrund steht. Somit ist es nicht möglich, aus diesen Modellen umfassende Aussagen über den Grad der Industrialisierung eines Unternehmens abzuleiten. Vielfach kann zwar evaluiert werden, inwieweit die jeweilige Organisation die vorgegebenen "Best Practices" erfüllt und somit einen bestimmten Reifegrad erreicht. Eine Be- wertung, angelehnt an die allgemeinen Prinzipien der Industrialisierung, ist dennoch nicht möglich, da keines der vorgestellten Prozessmodelle alle diese Grundsätze enthält. Zusammenfassend lässt sich feststellen, dass es in Literatur und Praxis bisher keinen Ansatz gibt, der den hier geforderten Anforderungen gerecht wird. 


\section{Grundlagen des IIM-Excellence- Modells}

\subsection{Konzepte des Excellence-Modells}

Das Excellence-Modell der Industrialisierung des Informationsmanagements (IIM) soll als Grundlage für Unternehmen dienen, um Funktionsbereiche/Rollen oder das gesamte Unternehmen bezüglich der Umsetzung von Grundgedanken der Industrialisierung zu überprüfen und gegebenenfalls Maßnahmen einzuleiten. Dabei dienen die verschiedenen Ausprägungen der Bewertungskriterien als Hilfsmittel für die Beschreibung von möglichen Wegen zur Weiterentwicklung des analysierten Bereiches. Hierfür unterliegt das entwickelte Rahmenwerk folgenden Voraussetzungen:

- Als vollständiges Rahmenwerk legt das Excellence-Modell die relevanten Bewertungsdimensionen zur Bestimmung des Industrialisierungsgrades fest.

- Zur Operationalisierung der definierten Dimensionen legt das Rahmenwerk in Abhängigkeit von der Analyseeinheit (siehe Abschnitt. 3.2) die Kriterien zur Bestimmung des Industrialisierungsgrades fest und beschreibt diese.

- Um das Grundkonzept des Excellence-Modells umzusetzen, werden für jedes Element Stufen beschrieben, die einen festgelegten Industrialisierungsgrad widerspiegeln.

- Basierend auf Erkenntnissen der Wissenschaft berücksichtigt das Rahmenwerk externe und interne Kontextfaktoren von Unternehmen oder Organisationseinheiten, um den Industrialisierungsgrad für das jeweilige Umfeld zu bestimmen. Dabei ist es für ein Unternehmen nicht wichtig, den höchsten Industrialisierungsgrad pro Element zu erzielen, sondern den optimalen für das jeweilige Umfeld.

- Das Rahmenwerk konzentriert sich ausschließlich auf die Gruppe der IT-Dienstleister - sowohl interne als auch externe.

\subsection{Analyseebenen}

In Anbetracht der Tatsache, dass IT-Dienstleister hoch komplizierte Unternehmen oder IT-Einheiten darstellen, sind für die Anwendbarkeit des im Folgenden beschriebenen Excellence-Modells die Analyseebenen zu spezifizieren. Analyseebenen sind abgeschlossene Untersuchungsbereiche, in denen festgelegte Eigenschaften überprüft werden.

Das "Big Picture» (Abb. 1) stellt die einzelnen Rollen im unternehmerischen Gesamtumfeld des Dienstleisters dar und legt die Grundlagen des Analyserasters. Dienstleister agieren in Analogie zu klassischen Industrieunternehmen innerhalb einer Lieferkette (Supply Chain) als Verarbeiter von Daten für Anwender auf der Kundenseite. Im Vordergrund der Betrachtungen steht stets die Prämisse, dass produzierte und abgegebene IT-Produkte des IT-Dienstleisters einen Nutzen beim Anwender erzeugen müssen, ohne den die Produktion ansonsten nicht sinnvoll wäre. Unter Berücksichtigung dieser Gedanken betreibt der IT-Dienstleister eine Lieferkette, beginnend mit der Beschaffung von benötigten Vorprodukten und Produktionsanlagen, der Produktion und endend mit der Auslieferung von IT-Produkten (Source, Make, Deliver) an den Anwender. Aufbauend auf diesem Rollen- und Aufgabenverständnis der klassischen Industrie lassen sich für IT-Dienstleister drei Analysebereiche identifizieren.

\section{Rollenspezifische Analyse}

Das abgebildete Modell (Abb. 1) gruppiert die Aufgaben eines IT-Dienstleisters in 12 Rollen wie beispielsweise Produktions-Engineering, Produkt-Engineering oder Produktmanagement. Innerhalb der Rollen werden Aufgaben, Ziele, Prozesse und Zielerreichungsindikatoren beschrieben.

\section{Prozessorientierte Analyse}

Die prozessorientierte Analyse untersucht entlang der Wertketten des Supply-Chain Council ${ }^{1}$ [Supply-Chain Council 2004; 2006a; 2006b] - 


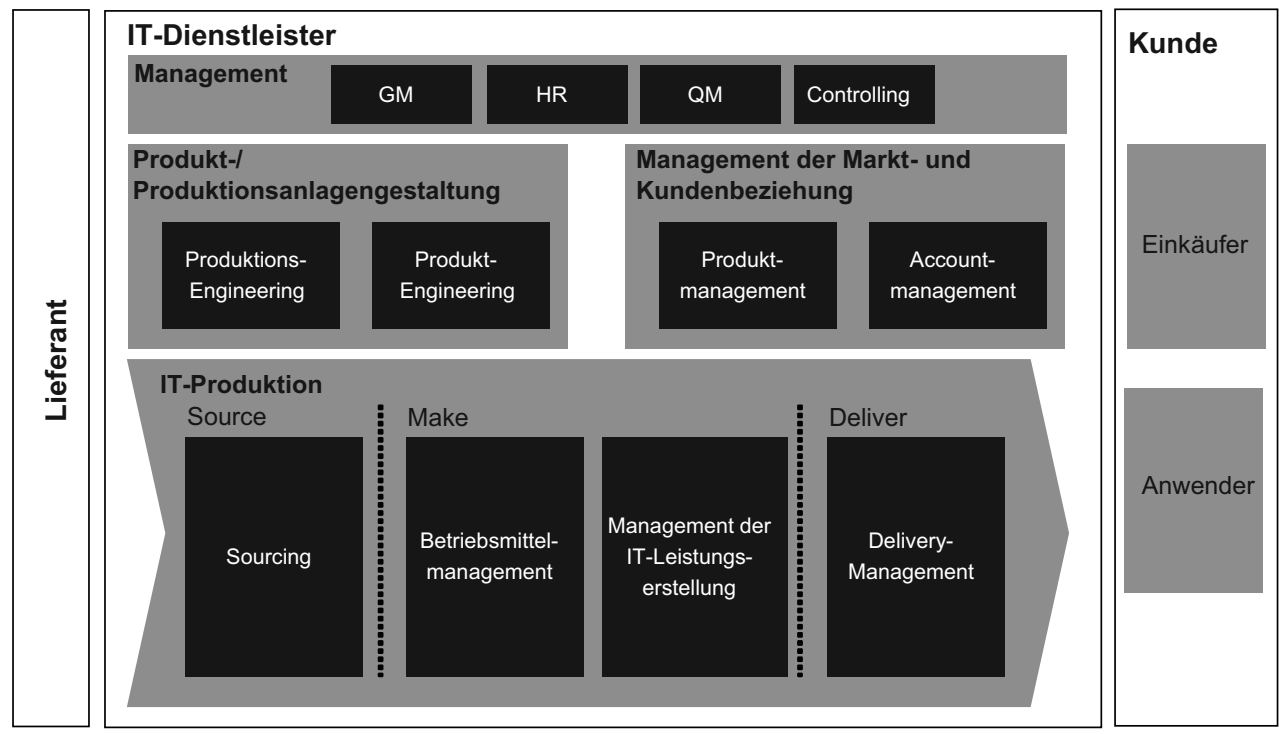

Abb. 1: Rollenmodell eines industrialisierten IT-Dienstleisters (in Anlehnung an [Zarnekow et al. 2006])

SCOR (Produktionsmanagement), DCOR (Produkt- und Produktionsgestaltung) und CCOR (Markt- und Kundenbeziehung) - Rollen, die die Aufgabenbereiche eines industrialisierten Dienstleisters beschreiben:

- General Management (GM): Vorgabe strategischer Ziele an die Geschäftsbereiche sowie permanente Überwachung und Steuerung der Umsetzung von Zielvorgaben

- Produkt- und Produktionsgestaltung: Planung und Umsetzung von IT-Produkten (klassische Softwareentwicklung) sowie Planung und Gestaltung der für die Produktion notwendigen Produktionsanlagen (Server, Speicher, Netze usw.)

- Markt- und Kundenbeziehung: Aufnahme von produktspezifischen Kundenanforderungen, Konfiguration von Produkten, Kommunikation des Produktnutzens gegenüber dem

1. SCOR (supply-chain reference model); DCOR (design-chain reference model); CCOR (customerchain reference model).
Anwender und Kunden sowie Pflege von Kundenkontakten und Marktpotenzialanalysen

- IT-Produktion: Management der Betriebsmittel wie Server, Speicher, Netze und Anwendungen; Management der Leistungserstellung zur Sicherstellung der Effizienz des Produktionsprozesses; Delivery-Management sowie das Management der Beschaffung von Betriebsmitteln und Daten, die in den Produktionsprozess eingehen.

- Querschnittsaufgaben: Controlling als Instanz zur Datenerhebung und Auswertung für das General Management und Qualitätsmanagement (OM)

\section{Gesamtunternehmen als Analyseebene}

Die höchste Aggregationsstufe des ExcellenceModells stellt das Gesamtunternehmen selbst dar. Diese komplexeste Variante untersucht sowohl auf Rollen- als auch Prozessbasis den Industrialisierungsgrad eines IT-Dienstleisters. Notwendige Voraussetzung für diese Analyseebene ist das erfolgreiche Bewerten der Einzelmodule. 


\subsection{Ergebnisraum}

Das IIM-Excellence-Modell ist in dieser Ausarbeitungsstufe explizit nicht als Reifestufenmodell konzipiert worden. Im Gegensatz zu Reifestufenmodellen wird als Ergebnis der Untersuchung kein aggregierter numerischer Wert in Form von "Sie haben die Stufe 4 (Anspruchsvoll) erreicht « errechnet. Stattdessen fokussiert sich das Excellence-Modell auf die Bewertung von einzelnen Kriterien bezüglich der Dimensionen Methode, Prozess und Assessment. Somit entsteht für einen bewerteten IT-Dienstleister ein Kontinuum an Einzelreifegraden, die problemund analysespezifisch Schwachstellen oder Stärken ausweisen.

\section{Aufbau des IIM-Excellence-Modells}

Das IIM-Excellence-Modell unterscheidet die Bestandteile Kategorie, Industrialisierungslevel, Dimension sowie Bewertungselement. Letzteres wird spezifisch für das jeweilige Analyseobjekt angewendet. Elementüberschneidungen zwischen einzelnen Modulen sind durchaus möglich und spiegeln gleiche Zielstellungen in unterschiedlichen Aufgabengebieten wider.

\subsection{Kategorien der Excellence}

Die Grundlage des Excellence-Modells bilden die vier Kategorien Produktorientierung, Prozessorientierung, Marktorientierung und integrierte Leistungserstellung. Ziel dieser ist die Operationalisierung der Fragestellung "Was wird unter Industrialisierung im Informationsmanagement verstanden?«. Ferner wurden die Kategorien explizit so gewählt, dass diese zunächst keinen thematischen Bezug zu einer Rolle oder einer Managementebene aufweisen, sondern zweckneutral, aber auf die Thematik "Industrialisierung" gerichtet sind. Durch diese Auswahl ist es möglich, für jedes Analyseobjekt (siehe Abschnitt 3.2) gezielt Messkriterien zu identifizieren, die wiederum den einzelnen Kategorien zugeordnet werden können.

Tabelle 2 stellt die vier grundlegenden Analysekategorien im Detail dar. Die Spalte "Perspektive" beschreibt mit einer kurzen Fragestellung den eingenommenen Blickwinkel auf den Themenaspekt.

\subsection{Stufen der Industrialisierung}

Die Idee des IIM-Excellence-Modells ist nicht, eine Aussage über "gut « oder »schlecht « zu geben. Stattdessen wird ein Ansatz verfolgt, der einen Raum an Ausprägungsmöglichkeiten der

\begin{tabular}{|l|l|l|}
\hline Kategorie & Perspektive & Beschreibung \\
\hline Produktorientierung & $\begin{array}{l}\text { Wie werden Absatz- und Fabrika- } \\
\text { tionsprodukte im Dienstleistungs- } \\
\text { unternehmen gemanagt? }\end{array}$ & $\begin{array}{l}\text { Industrialisierte Unternehmen managen ein Fabrika- } \\
\text { tions- und Absatzportfolio, um sowohl Effizienz-/ } \\
\text { Effektivitäts- als auch Markt- und Kundenanforderun- } \\
\text { gen gerecht zu werden. }\end{array}$ \\
\hline Prozessorientierung & $\begin{array}{l}\text { Wie standardisiert sind die Pro- } \\
\text { duktionsabläufe des Dienstleis- } \\
\text { ters? }\end{array}$ & $\begin{array}{l}\text { Industrialisierte Dienstleister produzieren ein hohes } \\
\text { Maß an Angebotsprodukten mithilfe standardisierter } \\
\text { Produktionsprozesse. }\end{array}$ \\
\hline Marktorientierung & $\begin{array}{l}\text { Wie werden Marktanforderungen } \\
\text { in den Betrieb integriert? }\end{array}$ & $\begin{array}{l}\text { Industrialisierte Unternehmen nehmen Marktanforde- } \\
\text { rungen schnell auf und setzen diese in inrer Produk- } \\
\text { tion um. }\end{array}$ \\
\hline $\begin{array}{l}\text { Integrierte } \\
\text { Leistungserstellung }\end{array}$ & $\begin{array}{l}\text { Wie arbeiten Aufgabenbereiche } \\
\text { zusammen? }\end{array}$ & $\begin{array}{l}\text { Industrialisierte IT-Dienstleister integrieren ihre Auf- } \\
\text { gabenbereiche. So sollte beispielsweise die } \\
\text { Produktentwicklung eng mit dem Betrieb zusammen- } \\
\text { arbeiten. }\end{array}$ \\
\hline
\end{tabular}

Tab. 2: Grundfragen der Industrialisierung des Informationsmanagements 
jeweiligen Analyseelemente zulässt. Innerhalb dieses Ausprägungsraums können sich Unternehmen mit ihren Aufgabenbereichen bewegen. Die Stufen an sich - eins, zwei, drei ... - geben daher keine Auskunft darüber, ob ein Optimum für das Unternehmen erreicht ist oder inwieweit eine Levelverbesserung anzustreben ist. So könnte beispielsweise die optimale Bewertungsstruktur für einen internen IT-Dienstleister eine andere Bewertungslinie aufweisen als für einen externen Dienstleister. Entscheidend ist die Hinterfragung der einzelnen Analyseergebnisse vor dem Hintergrund des bewerteten Unternehmens. So muss zum Beispiel ein gemäßigter Industrialisierungslevel für einen internen Dienstleister nicht zwingend bedeuten, dass ein höherer Level angestrebt werden soll. Sieht allerdings die Unternehmensstrategie eine Marktöffnung des Unternehmens vor, so sollte eine Überprüfung zur Anstrebung eines höheren Levels durchgeführt werden (Tab. 3 stellt die fünf Level kurz vor).

\subsection{Dimensionen der Analyseelemente}

Parallel zu den Kategorien und Industrialisierungslevel werden für jedes Analyseelement die Dimensionen Methode, Prozess und Assessment unterschieden.

Methode: Diese Dimension fokussiert auf den Einsatz von Methoden zur Unterstützung einer industrialisierten IT. So kann beispielsweise ein Produktkatalog auf Basis von CPU-Sekunden (interne Leistungseinheiten) [Nolan 1977], aber auch auf kundengeschäftsprozessorientierten Produkten (wie Transaktionen etc.) [Britzelmaier 1999] basieren. Beide Ausprägungen spiegeln einen bestimmten Industrialisierungslevel wider.

Prozess: Die Prozessdimension richtet sich auf die Messung derjenigen Industrialisierungslevel, die für die Umsetzung einer Methode im Unternehmen in Form von Prozessen relevant sind. Die Ausprägungen reichen von »nicht umgesetzt" bis »regelmäßige Durchführung". Demzufolge kann auch ein methodisch niedrig industrialisiertes Absatzportfolio einen hohen Industrialisierungsgrad in der Prozessdurchführung erreichen.

Assessment: In der Assessment-Dimension wird der Industrialisierungsgrad bezüglich der Überwachung des durchgeführten Prozesses gemessen. Ist beispielsweise eine hoch industrialisierte Methode konzipiert und umgesetzt, so kann die Bewertung der Monitoringprozesse sowohl eine hohe Industrialisierung (kontinuierliches Methoden- und Prozessmonitoring) als auch eine niedrige Industrialisierung (die eingesetzte Methode sowie die Prozesse werden nicht überwacht und verbessert) ausweisen.

\begin{tabular}{|l|l|l|}
\hline Nr. & Level & Beschreibung \\
\hline 1 & Nicht existent & Es sind keine Ansätze zur Industrialisierung vorhanden. \\
\hline 2 & Minimal & $\begin{array}{l}\text { Einige industrielle Konzepte sind erkennbar, die jedoch nur sporadisch Anwendung finden } \\
\text { (ad hoc). }\end{array}$ \\
\hline 3 & Gemäßigt & $\begin{array}{l}\text { Die Konzepte der Industrialisierung wurden verstanden und teilweise definiert. Tendenzen } \\
\text { zu festgelegten Prozessen sind vorhanden. }\end{array}$ \\
\hline 4 & Anspruchsvoll & $\begin{array}{l}\text { Ansätze der Industrialisierung sind implementiert. Nur wenige Konzepte fehlen zur voll- } \\
\text { ständigen Umsetzung. }\end{array}$ \\
\hline 5 & Voll industrialisiert & $\begin{array}{l}\text { Das höchste Maß an Industrialisierung bezüglich Strukturen, Methoden und Prozessen ist } \\
\text { erreicht. }\end{array}$ \\
\hline
\end{tabular}

Tab. 3: Fünf Industrialisierungslevel 


\section{Analyseelemente am Beispiel einer ausgewählten Rolle}

\subsection{Messkriterien der Industrialisierung am Beispiel des IT-Controllings}

Für die Rolle IT-Controlling wurden gemeinsam mit unseren Forschungspartnern insgesamt 14 Messkriterien in den vier beschriebenen Kategorien - Produktorientierung, Prozessorientierung, Marktorientierung sowie integrierte Leistungserstellung - identifiziert [Greenbaum 1988]. Gemäß den vier Kategorien wurden Messkriterien ausgewählt, die eine möglichst objektive Bestimmung des Industrialisierungsgrades erlauben:

\section{a. Produktorientierung}

- Absatzportfolio: Prozesse und Methoden zum Aufbau, zur Einführung und Kontrolle von Absatzportfolios. Absatzportfolios beschreiben die Schnittstelle zwischen ITDienstleister und Kunden der IT [Britzelmaier 1999; Uebernickel et al. 2006].

- Fabrikationsportfolio: Entkopplung der Absatz- und Fertigungssicht durch das Fabrikationsportfolio. Die Produktion kann standardisiert aufgebaut werden und somit eine transparente Kostenrechnung ermöglichen [Uebernickel et al. 2007].

- Produktkosten: Prozess zur Ermittlung, Steuerung und Kontrolle von Produktkosten. Der Ausweis von Produktkosten ermöglicht die gezielte Kontrolle von Produktions- und Absatzbereichen.

- Kosten der Fabrikationsprodukte: Prozess zur Ermittlung, Steuerung und Kontrolle von Fabrikationsproduktkosten. Ausweis ermöglicht die gezielte Kostenkontrolle der Produktionsprozesse.

- Kundenauftragsrechnung: Prozess zur Ermittlung, Steuerung und Kontrolle von kundenspezifischen Auftragskosten

b. Prozessorientierung

- Betriebsdatenerfassung: Prozess zur systematischen und kontinuierlichen Erfassung notwendiger Betriebsdaten
- Planung: Prozess zur Durchführung der regelmäßigen Budgetierung und Kostenplanung

- Ergebnisrechnung: Prozess zur Ermittlung des Unternehmenserfolges sowie der Produkt- und Kundenerfolgsrechnung

- Leistungsverrechnung: Prozess zur Durchführung der Verrechnung von gelieferten Leistungen an den Kunden

c. Marktorientierung

- Zielkosten: Methoden und Prozesse zur Berücksichtigung von Marktpreisen und -kosten in der Steuerung des IT-Dienstleisters

d. Integrierte Leistungserstellung

- Kapazitätskosten: Methoden und Prozesse zur Ermittlung und Auswertung von Kapazitätskosten (Leerkosten)

- Qualitätskosten: Methoden und Prozesse zur Bestimmung und Auswertung von Qualitätskosten (Kosten der Qualität und Nicht-Qualität)

\subsection{Praxisbeispiel}

Das folgende Praxisbeispiel wurde über einen Zeitraum von vier Monaten gemeinsam mit einem internen IT-Dienstleister im deutschsprachigen Raum erarbeitet. Die IT-Organisation beschäftigt derzeit circa 600 Mitarbeiter und deckt das Aufgaben- und Angebotsspektrum eines Full-Service-Providers - vom Innovator des Fachbereiches bis hin zum Alleinlieferanten vollständig ab. Auf Basis des dargestellten Excellence-Modells wurden spezifisch für die Funktion Controlling alle zuvor spezifizierten Messkriterien bewertet. Abbildung 2 zeigt am Beispiel von drei Messkriterien der Kategorie Produktorientierung die ausgearbeiteten Ergebnisse.

Folgende Erkenntnisse und Anregungen konnten durch Einsatz des Excellence-Modells gewonnen werden:

1. Es wurde eine Überprüfung initiiert, inwieweit der Aufbau von Prüfprozessen in Teilbereichen der Kostenrechnung eine steti- 


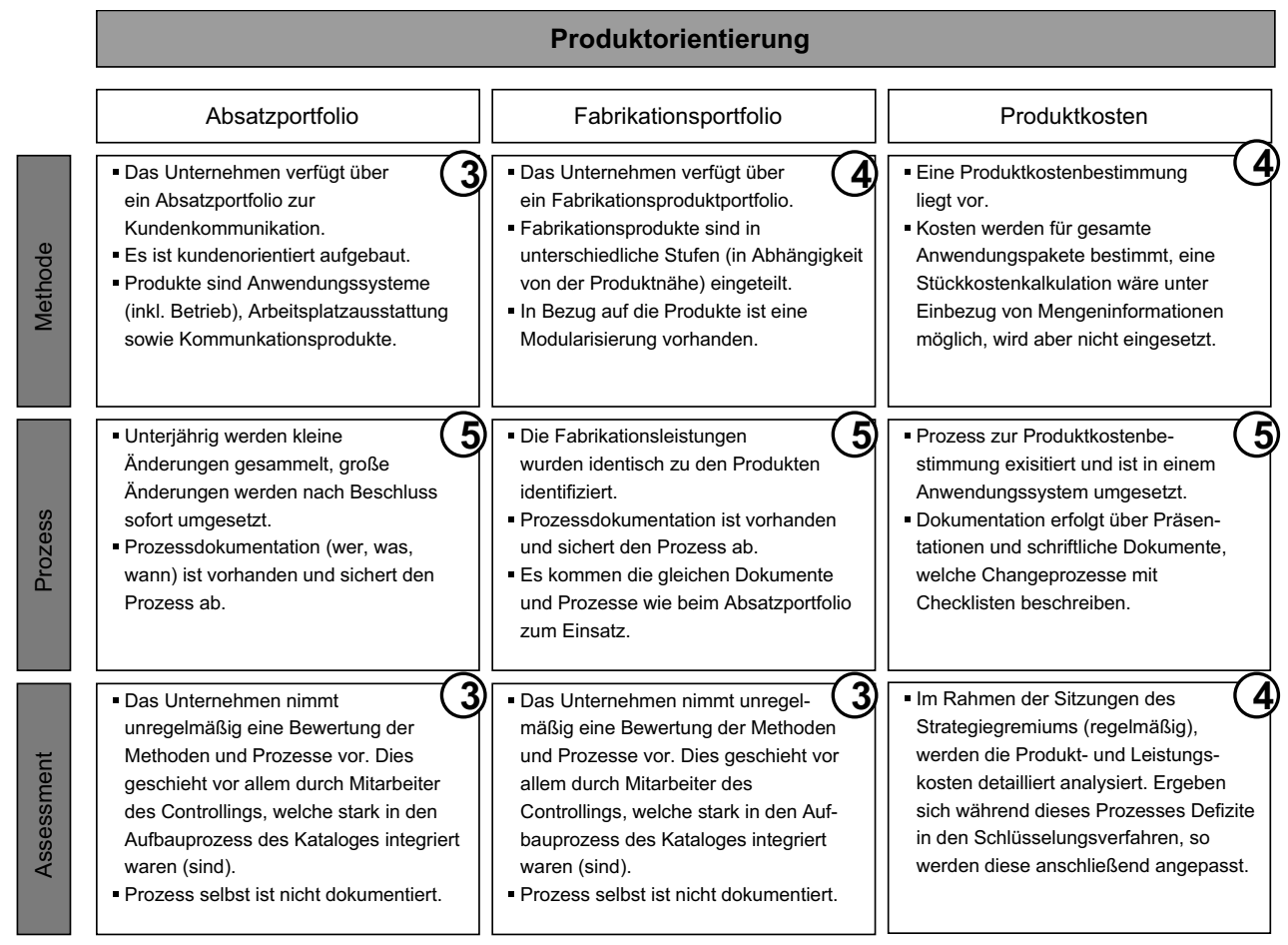

Abb. 2: Detailanalyse der Kategorie Produktorientierung am Beispiel eines deutschen IT-Dienstleisters (Kreise geben den Level an)

ge Fortentwicklung des Steuerungskonzepts sicherstellt.

2. Die Sicht »Integrierte Leistungserstellung" wurde miteinbezogen, indem verstärkt auch Informationsbedürfnisse der IT-Produktion, Entwicklung, des Qualitätsmanagements oder Supports stärker berücksichtigt werden. So könnten beispielsweise die Kosten nicht genutzter Kapazität oder mangelhafter Qualität bestimmt werden, um den produzierenden Einheiten weitere Steuerungsinformationen bereitzustellen.

3. Über Märkte ermittelte Zielkosten sollten zumindest auf Produktebene für nicht kundenindividuelle Produkte (Kommunikation, Arbeitsplatzausstattung) eingeführt und genutzt werden.
Wie das Beispiel deutlich gemacht hat, konnten Handlungsfelder für das Unternehmen aufgezeigt werden. Dennoch müssen zukünftig weitere Anwendungsfälle folgen, um einerseits die Module zu vervollständigen und andererseits die Gültigkeit der angewendeten Messkriterien nachzuweisen.

\section{Literatur}

[BSI 2002] British Standard Institute: BS 15000:2002 IT Service Management. London, 2002.

[Britzelmaier 1999] Britzelmaier, B.: Informationsverarbeitungs-Controlling - Ein datenorientierter Ansatz. Teubner, Stuttgart, Leipzig, 1999.

[Greenbaum 1988] Greenbaum, T.: The practical handbook and guide to focus group research. Lexington Books, London, 1988. 
[Hochstein 2006] Hochstein, A.: Planerische Prozesse eines industrialisierten Informationsmanagements. Dissertation. Universität St. Gallen, St. Gallen, 2006.

[Hochstein et al. 2006] Hochstein, A.; Uebernickel, F.; Brenner, W.: Operations Management in IS: Using the SCOR-Model to Source, Make and Deliver IS Services. 12th AMCIS, Acapulco, Mexico, 2006.

[Hyder et al. 2004] Hyder, E.; Heston, K.; Paulk, M.: The eSCM-SPv2: Model Overview, http://re ports-archive.adm.cs.cmu.edu/anon/isri2004/ CMU-ISRI-04-113.pdf; Zugriff am 15.12.2006.

[ISACA 2005] ISACA: Control Objectives for Information and Related Technology (CobiT), www. isaca.org/Content/NavigationMenu/ Members and Leaders/COBIT6/Obtain_COBIT/ Obtain_COBIT.htm; Zugriff am 14.12.2006.

[Niessink et al. 2005] Niessink F.; Clerc, V.; Tijdink T., van Vliet, H.: The IT Service Capability Maturity Model, wwwitservicecmm.org/doc/itscmm1.orc1.pdf; Zugriff am 15.12.2006.

[Nolan 1977] Nolan, R. L.: Controlling the costs of data services. In: Harvard Business Review, 55. Jg., 1977, Nr. 4, S. 114-124.

[SEI 2006] Software Engineering Institute: CMMI for Development, Version 1.2, www.sei.cmu.edu/ pub/documents/o6.reports/pdf/o6troo8.pdf; Zugriff am 12.12.2006.

[Supply-Chain Council 2004] Supply-Chain Council: Customer-Chain Operations Reference-model (CCOR) Version 1.0. Supply-Chain Council Inc., Pittsburgh, 2004.

[Supply-Chain Council 2006a] Supply-Chain Council: Design-Chain Operations Reference-model (DCOR) Version 1.0. Supply-Chain Council Inc., Pittsburgh, 2006.
[Supply-Chain Council 2006b] Supply-Chain Council: Supply-Chain Operations Reference-model (SCOR) Version 8.o. Supply-Chain Council Inc., Pittsburgh, 2006.

[Uebernickel et al. 2006] Uebernickel, F.; Bravo-Sànchez, C.; Zarnekow, R.; Brenner, W.: IS ServiceEngineering: A process model for the development of IS services. EMCIS, Alicante, Spain, 2006.

[Uebernickel et al. 2007] Uebernickel, F.; Scheeg, J.; Brenner, W.: Produkt- und serviceorientiertes IT-Controlling am Fallbeispiel T-Systems ActiveBilling. In: HMD - Praxis der Wirtschaftsinformatik, 44. Jg., Heft 254, 2007, S. 39-49.

[Yin 2002] Yin, R. K.: Case Study Research: Design and Methods. 3. Aufl., Sage Publications, London, 2002.

[Zarnekow et al. 2006] Zarnekow, R.; Brenner, W.; Pilgram, U.: Integrated Information Management: Applying Successful Industrial Concepts in IT. Springer-Verlag, Berlin, 2006.

Dipl.-Wirtsch.-Inform. Falk Uebernickel

Dr. Axel Hochstein

Dipl.-Ing. Veit Schulz

Prof. Dr. Walter Brenner

Universität St. Gallen

Institut für Wirtschaftsinformatik

Müller-Friedberg-Str. 8

$\mathrm{CH}-9000$ St. Gallen

\{falk.uebernickel, axel.hochstein, veit.schulz, walter.brenner\}@unisg.ch

www.iwi.unisg.ch 